\title{
Detection and prevalence of inducible clindamycin resistance in staphylococci
}

\author{
Gurdal Yilmaz, Kemalettin Aydin, Serap Iskender, Rahmet Caylan \\ and Iftihar Koksal \\ Department of Infectious Diseases and Clinical Microbiology, Karadeniz Technical University \\ School of Medicine, 61080 Trabzon, Turkey
}

Correspondence

Gurdal Yilmaz

gurdalyilmaz53@hotmail.com

Received 6 June 2006

Accepted 23 October 2006

\begin{abstract}
Staphylococcus aureus and coagulase-negative staphylococci (CNS) are recognized as causing nosocomial and community-acquired infections in every region of the world. The resistance to antimicrobial agents among staphylococci is an increasing problem. Clindamycin (CL) is considered to be one of the alternative agents in these infections. This study demonstrates a simple, reliable method (double-disc diffusion test) for detecting inducible resistance to $\mathrm{CL}$ in erythromycin-resistance (ER-R) isolates of S. aureus and CNS. A total of $883(52.3 \%)$ isolates of S. aureus and $804(47.7 \%)$ isolates of CNS were selected from recent (2003-2005) clinical isolates recovered in the laboratory of the authors; duplicate isolates were not included. A total of $214(12.6 \%)$ S. aureus and 308 (18.3\%) CNS isolates were selected based on ER-R and CL sensitivity using standard National Committee for Clinical Laboratory Standards disc diffusion testing. A total of 1687 staphylococcal isolates were included, consisting of $27.5 \%$ meticillin-resistant $S$. aureus, $24.8 \%$ meticillin-sensitive $S$. aureus, $36.1 \%$ meticillin-resistant CNS and $11.6 \%$ meticillin-sensitive CNS isolates: $30.9 \%$ of staphylococcal isolates (214 S. aureus and 308 CNS) that were erythromycin resistant and CL sensitive were tested for inducible resistance using the D-test. A D-shaped zone around the $\mathrm{CL}$ was observed for $70.9 \%$ of staphylococcal isolates ( $81.8 \%$ of $S$. aureus isolates and $63.3 \%$ of CNS isolates) with an ER-R and a clindamycin-sensitive (CL-S) phenotype. The organism was positive for inducible clindamycin resistance (CL-R). There was a $21.9 \%$ level of inducible macrolide-lincosamide-streptogramin $B$ resistance phenotype among all the staphylococcal isolates. When the $S$. aureus and CNS strains among all the staphylococcal isolates were compared statistically, inducible CL-R in CNS strains was determined to be $23 \%$ more positive $(P=0.028$, odds ratio $0.77,95 \%$ confidence interval 0.61-0.98). When a statistical comparison was performed among ER-R but CL-S staphylococcal isolates inducible CL-R in $S$. aureus strains was determined to be 2.6 times more positive ( $P=0.000$, odds ratio $2.6,95 \%$ confidence interval 1.68-4.04). A simple, reliable method of detecting inducible resistance to $C L$ in ER-R isolates of $S$. aureus and CNS is described. Clinical microbiology laboratories should use the double-disc diffusion test as standard practice with all ER-R staphylococci. CL should not be used in patients with infections caused by inducibly resistant staphylococcal isolates. Therapeutic failures may thus be avoided.
\end{abstract}

\section{INTRODUCTION}

Staphylococcus aureus and coagulase-negative staphylococci (CNS) are recognized to be causing nosocomial and community-acquired infections in every region of world. The increasing prevalence of meticillin resistance among staphylococci is an increasing problem (Fokas et al., 2005).

Abbreviations: $\mathrm{Cl}$, confidence interval; $\mathrm{CL}$, clindamycin; CNS, coagulase-negative staphylococci; ER, erythromycin; MRCNS, meticillinresistant coagulase-negative staphylococci; MRSA, meticillin-resistant S. aureus; MSCNS, meticillin-sensitive coagulase-negative staphylococci; MSSA, meticillin-sensitive S. aureus; OR, odds ratio.
Once these organisms are recognized to be causing infections in a region, it is of interest to determine which of the alternatives to vancomycin are suitable for therapy. In vitro susceptibility to clindamycin (CL), trimethoprimsulfamethoxazole, erythromycin (ER), quinolone antibiotics and tetracyclines has frequently been reported (Hussain et al., 2000; Groom et al., 2001; Naimi et al., 2001). The macrolide-lincosamide-streptogramin $\mathrm{B}\left(\mathrm{MLS}_{\mathrm{B}}\right)$ family of antibiotics is commonly used in the treatment of staphylococcal infections (Fiebelkorn et al., 2003). However, one important issue in CL treatment is the risk of clinical failure during therapy. Therapeutic failures caused by $\mathrm{MLS}_{\mathrm{B}}$ inducible resistance are being more commonly reported. 
The MLS family of antibiotics has three different mechanisms of resistance: target site modification, enzymic antibiotic inactivation and macrolide efflux pumps (Goldman \& Capobianco, 1990; Leclercq \& Courvalin, 1991a, b). Inducible MLS $_{\mathrm{B}}$ resistance cannot be determined using standard susceptibility test methods, including standard broth-based or agar dilution susceptibility tests (Fiebelkorn et al., 2003). Low levels of ER are the most effective inducer of inducible $M L S_{B}$ resistance (Weisblum \& Demohn, 1969). Antimicrobial susceptibility data are important for the management of infections, but false susceptibility results may be obtained if isolates are not tested for inducible CL resistance (CL-R) (Fiebelkorn et al., 2003; Drinkovic et al., 2001).

This study demonstrates a simple, reliable and significant method (double-disc diffusion test) of detecting inducible resistance to CL in ER-resistance (ER-R) isolates of $S$. aureus and CNS.

\section{METHODS}

Staphylococcal isolates were selected from clinical isolates recovered in our laboratory from 2003 to 2005; duplicate isolates were not included. A total of 214 S. aureus and 308 CNS isolates were selected based on ER-R and CL sensitivity using standard National Committee for Clinical Laboratory Standards (NCCLS) disc diffusion testing. These isolates were tested for inducible resistance using the D-test. A 0.5 McFarland equivalent suspension of organisms was inoculated onto a Mueller-Hinton agar (MHA) plate as described in the NCCLS recommendations (National Committee for Clinical Laboratory Standards, 2003). CL $(2 \mu \mathrm{g})$ and ER $(15 \mu \mathrm{g})$ discs were placed $15 \mathrm{~mm}$ apart from the centre on the MHA. Plates were analyzed after $18 \mathrm{~h}$ of incubation at $35^{\circ} \mathrm{C}$. Interpretation of the diameters of zones of inhibition was as follows: ER sensitive (ER-S) $\geqslant 23 \mathrm{~mm}$, ER intermediate resistance $14-22 \mathrm{~mm}$, ER-R $\leqslant 13 \mathrm{~mm}$; clindamycin sensitive (CL-S) $\geqslant 21 \mathrm{~mm}$, CL intermediate resistance $15-20 \mathrm{~mm}$ and CL-R $\leqslant 14 \mathrm{~mm}$. If the ER zone is $\leqslant 13 \mathrm{~mm}$ and the CL zone is $\geqslant 21 \mathrm{~mm}$ and both have a circular shape, the organism is negative for inducible resistance (D-test negative). If the ER zone is $\leqslant 13 \mathrm{~mm}$ and the CL zone is $\geqslant 21 \mathrm{~mm}$ with a D-shaped zone around the CL, the organism is positive for inducible resistance (Dtest positive) (Fig. 1).

\section{RESULTS}

A total of 1687 staphylococcal isolates (883 S. aureus and 804 CNS) obtained from consecutive clinical specimens were included, consisting of $27.5 \%$ (464) meticillin-resistant $S$. aureus (MRSA), $24.8 \%$ (419) meticillin-sensitive S. aureus

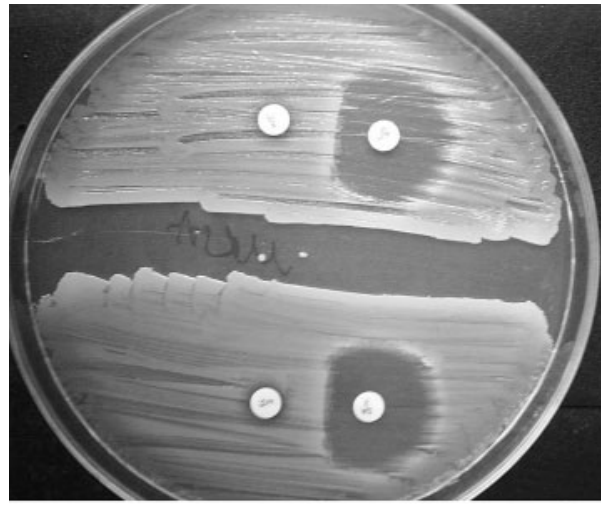

Fig. 1. Double-disc diffusion test (D-test) demonstrating ER disc induction of CL-R; a blunting of the zone of inhibition around the $\mathrm{CL}$ disk is produced that forms a $\mathrm{D}$ shape. The chi-square test was used to determine significant differences between categorical variables. $P<0.05$ was considered significant.

(MSSA), 36.1\% (608) meticillin-resistant CNS (MRCNS) and $11.6 \%$ (196) meticillin-sensitive CNS (MSCNS) isolates. Of the staphylococcal isolates, $49.8 \%$ had the CL$S$ phenotype, $28.3 \%$ the constitutive resistance phenotype and $21.9 \%$ the inducible resistance phenotype (Table 1 ). The constitutive resistance phenotype predominated over the susceptible phenotype and inducible resistance phenotype (44.2, 31.5 and $24.4 \%$, respectively) among the MRSA isolates. The CL-S phenotype predominated over the inducible resistance phenotype and constitutive resistance phenotype (80.7, 14.8 and $4.5 \%$, respectively) among MSSA isolates. The constitutive resistance phenotype predominated over the susceptible phenotype and inducible resistance phenotype (38.3, 36.0 and $25.7 \%$, respectively) among the MRCNS isolates. The CL-S phenotype predominated over the inducible resistance phenotype and constitutive resistance phenotype (69.9, 19.9 and 10.2\%, respectively) among the MSCNS isolates (Table 1).

While in meticillin-resistant staphylococcal isolates the constitutive CL-R phenotype level was $40.9 \%$ and the inducible resistance phenotype level $25.1 \%$, in meticillinsensitive staphylococcal isolates the constitutive CL-R phenotype level was $6.3 \%$ and the inducible resistance phenotype level $16.4 \%$. When the results were statistically compared in meticillin-resistant staphylococcal isolates the

Table 1. Susceptibility to $E R$ and $C L$ among all staphylococcal isolates

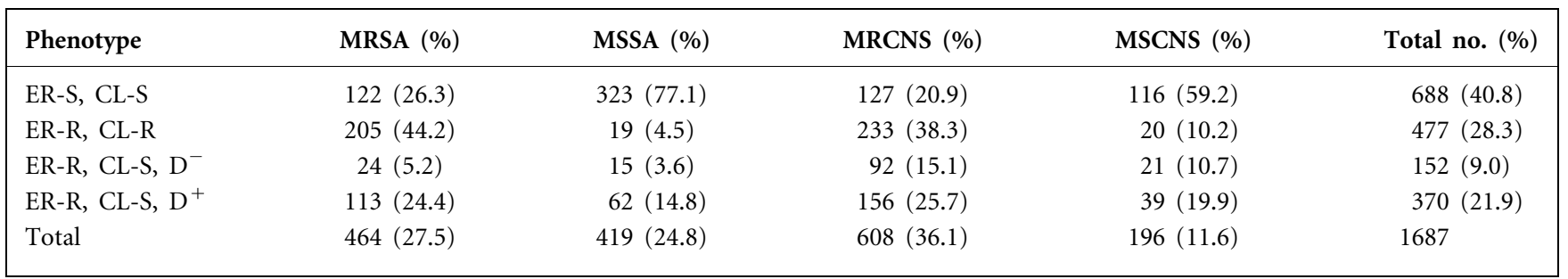


constitutive CL-R phenotype was determined to be 10.2 times greater $[P=0.000$, odds ratio $(\mathrm{OR}) 10.2,95 \%$ confidence interval (CI) 7.13-14.66] and the inducible resistance phenotype 1.7 times greater $(P=0.000$, OR 1.7; $95 \%$ CI 1.31-2.22) than that in meticillin-sensitive staphylococcal isolates.

There was a $21.9 \%$ inducible $\mathrm{MLS}_{\mathrm{B}}$ resistance phenotype level among all the staphylococcal isolates (19.8\% among S. aureus and $24.3 \%$ among CNS isolates). When the S. aureus and CNS strains among all staphylococcal isolates were statistically compared, inducible CL-R in CNS strains was determined to be $23 \%$ more positive $(P=0.028$, OR 0.77 , 95\% CI 0.61-0.98) (Table 2). MRSA and MSSA strains among $S$. aureus isolates were also statistically compared, and inducible CL-R in MRSA strains was determined to be 1.85 times more positive ( $P=0.000$, OR $1.85,95 \%$ CI $1.30-2.65)$.

A total of 522 (30.9\%) staphylococcal isolates (214 S. aureus and $308 \mathrm{CNS}$ ) that showed ER-R but were CL-S were tested for inducible resistance using the D-test. Three hundred and seventy $(70.9 \%)$ staphylococcal isolates $(81.8 \%$ of S. aureus and $63.3 \%$ of CNS) with the ER-R and CL-S phenotype were observed to have a D-shaped zone around the CL. The organism was positive for inducible CL-R. When a statistical comparison was performed among the ER-R but CL-S staphylococcal isolates, inducible CL-R in $S$. aureus strains was determined to be 2.6 times more positive ( $P=0.000$, OR 2.6, $95 \%$ CI 1.68-4.04).

\section{DISCUSSION}

The increasing frequency of staphylococcal infections among patients and changing patterns in antimicrobial resistance have led to renewed interest in the use of CL therapy to treat such infections (Frank et al., 2002). CL is frequently used to treat skin and bone infections because of its tolerability, cost, oral form and excellent tissue penetration, and the fact that it accumulates in abscesses and no renal dosing adjustments are needed (Kasten, 1999). Good oral absorption makes it an important option in outpatient therapy or as follow-up after intravenous therapy. CL is a good alternative for the treatment of both meticillin-resistant and -susceptible staphylococcal infections (Fiebelkorn et al., 2003). CL-R can develop in

Table 2. Susceptibility to $E R$ and $C L$ in $S$. aureus and CNS strains

\begin{tabular}{|lcccccc|}
\hline Phenotype & S. aureus (\%) & CNS (\%) & $\boldsymbol{P}$ & OR & 95\% CI \\
\hline ER-S, CL-S & $445(50.4)$ & $243(30.2)$ & 0.000 & 2.35 & $1.91-2.88$ \\
ER-R, CL-R & $224(25.4)$ & $253(31.5)$ & 0.005 & 0.74 & $0.60-0.98$ \\
ER-R, CL-S, D & $39(4.4)$ & $113(14.1)$ & 0.000 & 0.28 & $0.19-0.42$ \\
ER-R, CL-S, D & $175(19.8)$ & $195(24.3)$ & 0.028 & 0.77 & $0.61-0.98$ \\
Total & 883 & 804 & - & - & - \\
\hline
\end{tabular}

staphylococcal isolates with the inducible phenotype, and spontaneous constitutively resistant mutants have been selected from such isolates both in vitro and in vivo during CL therapy (Drinkovic et al., 2001; Panagea et al., 1999; Siberry et al., 2003). In 1969, McGehee et al. demonstrated the development of CL-R in vivo and in vitro in ER-resistant staphylococci (McGehee et al., 1969). Other authors have confirmed the rapid in vitro conversion of inducible to constitutive $\mathrm{MLS}_{\mathrm{B}}$ resistance in staphylococci (Panagea et al., 1999; Werckenthin et al., 1999). The 2004 NCCLS guidelines recommend use of the double-disc test and suggest that isolates with the inducible resistance phenotype should be reported as CL resistant (National Committee for Clinical Laboratory Standards, 2004). However, the clinical efficacy of CL treatment for infections caused by inducibly resistant staphylococci remains unclear. The few cases reported so far have presented conflicting results, and the elimination of a potentially useful drug such as CL is undesirable, especially for the treatment of MRSA infections (Rao, 2000).

This study demonstrates that the D shape of the CL zone adjacent to an ER disc in a conventional disc diffusion test can serve to detect $S$. aureus or CNS strains with inducible resistance to CL. A total of $81.8 \%$ of S. aureus and $63.3 \%$ of CNS isolates with the ER-R and CL-S phenotype demonstrated inducible resistance. Overall, the results indicate a high incidence $(21.9 \%)$ of the inducible MLS $_{\mathrm{B}}$ resistance phenotype among all the staphylococcal isolates ( $19.8 \%$ of $S$. aureus and $24.3 \%$ of CNS isolates). Our results demonstrate that in the case of a staphylococcal infection inducible $M_{\mathrm{B}}$ resistance in CNS strains is greater than that in S. aureus strains. When the clinician is faced with a staphylococcal isolate, the probability that this strain may be inducible $M S_{B}$ resistant is $21.9 \%$. If this strain is CNS, then the probability that this strain may be inducible $\mathrm{MLS}_{\mathrm{B}}$ resistant will be $20.3 \%$ greater. Provided that this strain is meticillin resistant, the possibility that the strain may be inducible $M L S_{B}$ resistant is 1.7 times higher compared to a meticillin-sensitive strain. Provided that the clinician is informed of ER-R and CL-S phenotype staphylococcal isolates, the probability of inducible $\mathrm{MLS}_{\mathrm{B}}$ resistance is high. If it is an S. aureus strain, its inducible $\mathrm{MLS}_{\mathrm{B}}$ resistance is 2.6 times higher than that of CNS strains. In such a case, the doctor is advised to provide treatment considering inducible $M L S_{B}$ resistance.

In a study conducted in Brazil, $11.3 \%$ of $S$. aureus and $13.7 \%$ of CNS isolates were determined to have the inducible $\mathrm{MLS}_{\mathrm{B}}$ resistance phenotype (Van der Heijden et al., 2001). It was reported that $80 \%$ of S. aureus and $63 \%$ of CNS isolates with the ER-R and CL-S phenotype demonstrated inducible resistance in a New York City medical centre (Van Horn \& Toth, 2003). Fiebelkorn concluded that $29 \%$ of 114 ER-R S. aureus isolates demonstrated inducible CL-R while $30 \%$ of CNS isolates demonstrated inducible CL-R (Fiebelkorn et al., 2003).

In our study, the inducible CL-R phenotype level was $24.4 \%$ among MRSA isolates, $14.8 \%$ among MSSA isolates, $25.7 \%$ 
among MRCNS isolates and 19.9\% among MSCNS isolates. In another study conducted in Turkey, $5.7 \%$ among MRSA isolates, $3.6 \%$ of MSSA isolates, $30.8 \%$ of MRCNS isolates and $15.3 \%$ of MSCNS isolates were determined to have the inducible CL-R phenotype (Azap et al., 2005). Schreckenberger et al. (2004) reported incidences of inducible CL-R of $7-12 \%$ for MRSA, $19-20 \%$ for MSSA and 14-35\% for CNS in two hospitals.

In the light of the restricted range of antibiotics available for the treatment of meticillin-resistant staphylococcal infections and the known limitations of vancomycin, CL should be considered for the management of serious soft tissue infections with meticillin-resistant staphylococci that either are sensitive to CL or exhibit inducible CL-R (Rao, 2000).

The double-disc diffusion test is necessary to correctly discriminate between inducible CL-R and susceptibility to CL. However, if inducible resistance can be reliably detected on a routine basis in clinically significant isolates, CL can be safely and effectively used in those patients with true CL-S strains. ER-R staphylococci should not be assumed to have CL-R.

In this study, we describe a simple, reliable method of detecting inducible resistance to CL in ER-R isolates of $S$. aureus and CNS. Clinical microbiology laboratories should use the double-disc diffusion test as standard practice with all ER-R staphylococci. Furthermore, in applying the susceptibility test to staphylococcal isolates, clinical microbiology laboratories should place the ER disc $15 \mathrm{~mm}$ apart from the CL disc. Thanks to the early detection of inducible $\mathrm{MLS}_{\mathrm{B}}$ resistance, such a measure will enable the clinician to save time. Consequently, treatment using CL can be omitted in patients with infections caused by inducibly resistant strains, and therapeutic failures may thus be avoided.

\section{REFERENCES}

Azap, O. K., Arslan, H., Timurkaynak, F., Yapar, G., Oruc, E. \& Gagir, U. (2005). Incidence of inducible clindamycin resistance in staphylococci: first results from Turkey. Clin Microbiol Infect 11, 582-584.

Drinkovic, D., Fuller, E. R., Shore, K. P., Holland, D. J. \& Ellis-Pegler, R. (2001). Clindamycin treatment of Staphylococcus aureus expressing inducible clindamycin resistance. J Antimicrob Chemother 48 , 315-316.

Fiebelkorn, K. R., Crawford, S. A., McElmeel, M. L. \& Jorgensen, J. H. (2003). Practical disk diffusion method for detection of inducible clindamycin resistance in Staphylococcus aureus and coagulasenegative staphylococci. J Clin Microbiol 41, 4740-4744.

Fokas, S., Fokas, S., Tsironi, M., Kalkani, M. \& Dionysopouloy, M. (2005). Prevalence of inducible clindamycin resistance in macrolideresistant Staphylococcus spp. Clin Microbiol Infect 11, 337-340.

Frank, A. L., Marcinak, J. F., Mangat, P. D., Tjhio, J. T., Kelkar, S., Schreckenberger, P. C. \& Quinn, J. P. (2002). Clindamycin treatment of methicillin-resistant Staphylococcus aureus infections in children. Pediatr Infect Dis J 21, 530-534.

Goldman, R. C. \& Capobianco, J. O. (1990). Role of an energydependent efflux pump in plasmid pNE24-mediated resistance to
14- and 15-membered macrolides in Staphylococcus epidermidis. Antimicrob Agents Chemother 34, 1973-1980.

Groom, A. V., Wolsey, D. H., Naimi, T. S., Smith, K., Johnson, S., Boxrud, D., Moore, K. A. \& Cheek, J. E. (2001). Community-acquired methicillin-resistant Staphylococcus aureus in a rural American Indian community. JAMA 286, 1201-1205.

Hussain, F. M., Boyle-Vavra, S., Bethel, C. D. \& Daum, R. S. (2000). Current trends in community-acquired methicillin-resistant Staphylococcus aureus at a tertiary care pediatric facility. Pediatr Infect Dis J 19, 1163-1166.

Kasten, M. J. (1999). Clindamycin, metronidazole, and chloramphenicol. Mayo Clin Proc 74, 825-833.

Leclercq, R. \& Courvalin, P. (1991a). Bacterial resistance to macrolide, lincosamide, and streptogramin antibiotics by target modification. Antimicrob Agents Chemother 35, 1267-1272.

Leclercq, R. \& Courvalin, P. (1991b). Intrinsic and unusual resistance to macrolide, lincosamide, and streptogramin antibiotics in bacteria. Antimicrob Agents Chemother 35, 1273-1276.

McGehee, R. F., Barret, F. F. \& Finland, F. (1969). Resistance of Staphylococcus aureus to lincomycin, clinimycin, and erythromycin. Antimicrob Agents Chemother 13, 392-397.

Naimi, T. S., LeDell, K. H., Boxrud, D. J., Groom, A. V., Steward, C. D., Johnson, S. K., Besser, J. M., O'Boyle, C., Danila, R. N. \& other authors (2001). Epidemiology and clonality of community-acquired methicillin-resistant Staphylococcus aureus: Minnesota, 1996-98. Clin Infect Dis 33, 990-996.

National Committee for Clinical Laboratory Standards (2003). Performance Standards for Antimicrobial Disk Susceptibility Tests, approved standard M2-A8. Wayne, PA: National Committee for Clinical Laboratory Standards.

National Committee for Clinical Laboratory Standards (2004). Performance Standards for Antimicrobial Disk Susceptibility Testing, fourteenth informational supplement, document M100-S14. Wayne, PA: National Committee for Clinical Laboratory Standards.

Panagea, S., Perry, J. D. \& Gould, F. K. (1999). Should clindamycin be used in treatment of patients with infections caused by erythromycin-resistant staphylococci? J Antimicrob Chemother 44, 581-582.

Rao, G. G. (2000). Should clindamycin be used in treatment of patients with infections caused by erythromycin-resistant staphylococci? J Antimicrob Chemother 45, 715.

Schreckenberger, P. C., llendo, E. \& Ristow, K. L. (2004). Incidence of constitutive and inducible clindamycin resistance in Staphylococcus aureus and coagulase-negative staphylococci in a community and a tertiary care hospital. J Clin Microbiol 42, 2777-2779.

Siberry, G. K., Tekle, T., Carroll, K. \& Dick, J. (2003). Failure of clindamycin treatment of methicillin-resistant Staphylococcus aureus expressing inducible clindamycin resistance in vitro. Clin Infect Dis 37, $1257-1260$.

Van der Heijden, I. M., Sinto, S., Oplustil, C. \& Mendes, C. (2001). In Abstracts of the101st General Meeting of the American Society for Microbiology 2001, abstract A-86. Washington, DC: American Society for Microbiology.

Van Horn, K. G. \& Toth, C. (2003). In Abstracts of the 103rd General Meeting of the American Society for Microbiology 2003, abstract C074. Washington, DC: American Society for Microbiology.

Weisblum, B. \& Demohn, V. (1969). Erythromycin-inducible resistance in Staphylococcus aureus: survey of antibiotic classes involved. J Bacteriol 98, 447-452.

Werckenthin, C., Schwarz, S. \& Westh, H. (1999). Structural alterations in the translational attenuator of constitutively expressed ermC genes. Antimicrob Agents Chemother 43, 1681-1685. 